\title{
Solapamiento de la dieta de Passer domesticus y Zonotrichia capensis en un agroecosis- tema de la costa central del Perú
}

\section{Diet overlap of the invasive House Sparrow and the native Rufous-collared Sparrow in an agroecosystem of Central Coast of Peru}

\section{Katherine Menacho ${ }^{1}$, Letty Salinas* ${ }^{* 1,2}$ y César Arana ${ }^{1,3}$}

1 Facultad de Ciencias Biológicas, Universidad Nacional Mayor de San Marcos

2 Departamento de Ornitología, Museo de Historia Natural, Universidad Nacional Mayor de San Marcos

3 Departamento de Ecología, Museo de Historia Natural, Universidad Nacional Mayor de San Marcos

*Autor de correspondencia

Email Katherine Menacho: katymj_1008@hotmail.com

Email Letty Salinas: Isalinass@unmsm.edu.pe

Email César Arana: caranab@unmsm.edu.pe

\begin{abstract}
Resumen
Se analizó el solapamiento de las dietas de dos especies de gorriones en un agroecosistema de la costa central del Perú, una de las especies es invasora (Passer domesticus), mientras que la otra es nativa (Zonotrichia capensis). El presente trabajo muestra que a pesar de que $Z$. capensis tiene una dieta más diversa $\left(H^{\prime}=2.07\right)$ que $P$. domesticus $\left(H^{\prime}=1.63\right)$, presentan alta similitud entre ellas $\left(D_{\max }=0.15, p>0.05\right)$. El índice de Schoener fue $\phi=0.73$ y junto con en el análisis de escalamiento multidimensional no métrico muestran un amplio solapamiento en la dieta de ambos gorriones en los diferentes meses de evaluación. Estos resultados permiten concluir que existe un potencial impacto de la invasión de Passer domesticus sobre la especie nativa Zonotrichia capensis por presentar un marcado solapamiento del alimento.
\end{abstract}

Palabras clave: Especies invasoras; Ica; nicho trófico; solapamiento de nicho; Aves.

\section{Abstract}

We analyse the diet overlap of two species of sparrows in an agroecosystem of the Central Coast of Peru, one of the species is invasive House Sparrow, while the other is native Rufous-collared Sparrow. We show that although native species has a more diverse diet $\left(H^{\prime}=2.07\right)$, than invasive species $\left(H^{\prime}=1.63\right)$, they present high similarity between them $\left(D_{\text {max }}=0.15, p>0.05\right)$. The Schoener index was $\phi=0.73$ and together with the non-metric multidimensional scaling analysis they show a broad diet overlap of both sparrows in the different months of evaluation. These results allow to conclude that there is a potential impact of the invasion of House Sparrow on the native species Rufous-collared Sparrow by food overlap.

Keywords: Zonotrichia capensis; Passer domesticus; Ica; niche overlap; birds.

\section{Citación:}

Menacho K., L. Salinas y C. Arana. 2018. Solapamiento de la dieta de Passer domesticus y Zonotrichia capensis en un agroecosistema de la costa central del Perú. Revista peruana de biología 25(2): 111 - 116 (Mayo 2018). doi: http://dx.doi.org/10.15381/rpb.v25i2.14687

Fuentes de financiamiento: Proyectos de Investigación VRIPUNMSM 071001161, 071001291, 081001291, 091001171, 101001271 $101001281,111001221,111001115,121001161,121001191$

$\begin{array}{ll}\text { Presentado: } & 09 / 06 / 2017 \\ \text { Aceptado: } & 28 / 02 / 2018\end{array}$

Permisos de colecta: Servisio Nacional Forestal y de Fauna Silvestre (SERFOR) del Ministerio de Agricultura y Riego - permisos de investigación RD 0263-2014-MINAGRI-DGFFS/DGEFFS, RD 0267-2014-MINAGRI-DGFFS/DGEFFS, Autorización 051-2005-INRENAIFFS-DCB.

Información sobre los autores: KM realizó los análisis e identificación de la mayoría de las muestras y preparó el primer borrador del manuscrito, CA y LS realizaron el diseño del muestreo y las evaluaciones de campo, participaron en el análisis de la información y en la elaboración del manuscrito.

Los autores no incurren en conflictos de intereses. 


\section{Introducción}

La competencia que involucra especies invasoras es particularmente importante debido a que puede causar la extinción de especies nativas (Gurnell et al. 2004). Esto es más preocupante, si se toma en cuenta que la introducción no controlada de especies exóticas, es considerada una de las mayores amenazas a la biodiversidad mundial, siendo señalada actualmente como la segunda causa de extinción de especies, después de la destrucción de hábitats (Zaret \& Paine 1973, Vitousek 1990, Lambertini et al. 2011).

En el caso de las aves, una de las especies invasoras con mayor éxito es el gorrión Passer domesticus (Linnaeus, 1758), natural de Europa y el Norte de África, que actualmente ha ampliado su distribución a todos los continentes con excepción de la Antártida (Anderson 2006). Esta ave llegó a Norteamérica entre 1852-1853 y para 1915 ya había colonizado Canadá y Estados Unidos, continuando su avance a través de México, Guatemala, Costa Rica y Panamá (Anderson 2006, MacGregor et al. 2009, Moulton et al. 2010). En Sudamérica existieron múltiples puntos de introducción: en Buenos Aires en 1872, en Santiago de Chile en 1904 y en Río de Janeiro en 1905; desde entonces su presencia y avance ha sido reportada frecuentemente (Baker 1995). Al Perú, llegó proveniente de Chile, donde varios individuos fueron liberados en Santiago en 1904 (Baldini \& Pancel 2002, Anderson 2006). Para 1924 alcanzó Antofagasta, en 1940 el límite norte de Chile, y en 1951 fue registrada con frecuencia en Tacna e Ilo; alcanzando luego Lima y avanzando hacia la costa norte (Koepcke 1952, 1964).

En la actualidad $P$. domesticus se encuentra distribuido en toda la costa del Perú, tanto en áreas urbanas como rurales (Gonzáles 2004, Salinas 2007, Salinas et al. 2007, Takano \& Castro 2007, Angulo et al. 2010, Schulenberg et al. 2010, Pulido et al. 2013, Salinas et al. 2013). Las posibles consecuencias de su introducción en nuestro país, aún no han sido estudiadas, sin embargo, estudios en otros países muestran que la presencia de $P$. domesticus es capaz de modificar de manera significativa las comunidades de los ecosistemas invadidos, al competir eficientemente por los recursos ocasionando la disminución de poblaciones de aves nativas (Anderson 2006, MacGregor et al. 2009, Feare 2010). Estos resultados podrían estar relacionados con el comportamiento agresivo observado en $P$. domesticus, que ataca constantemente a las especies nativas en sitios de forrajeo; así como con su gran capacidad de explotar recursos sobre todo en áreas cercanas a poblados y ciudades (Kalinoski 1975, Gowaty 1981, MacGregor et al. 2009).

En particular, Goodall (1946) y Koepcke (1964) consideraron que $P$. domesticus, podría afectar al gorrión nativo Zonotrichia capensis (Statius Muller, 1776) y a otras aves que se alimentan de semillas, debido a que compartirían parcialmente el nicho, lo que podría causar un desequilibrio en sus poblaciones. A esto se le debe añadir que, las ciudades, poblados y agroecosistemas de la costa peruana ofrecen un ambiente favorable para especies exóticas, en especial a las que se alimentan de semillas (Chace \& Walsh 2006), lo que podría otorgar mayores ventajas al gorrión invasor. Passer domesticus tiene un elevado éxito al invadir agroecosistemas convirtiéndose en una auténtica plaga, que consume grandes cantidades de semillas de los cultivos y las malezas, además de insectos (Torres-Vila et al. 2015, Singh et al. 2016), afectando la avifauna local (Sax et al. 2000, Anderson 2006). En el presente estudio se evalúa la existencia de solapamiento en la dieta del gorrión invasor $P$. domesticus y la especie nativa $Z$. capensis en un agroecosistema de Ica.

\section{Materiales y métodos}

Área de Estudio.- El área de estudio se encuentra en el departamento de Ica, en el fundo "La Catalina" (14 ${ }^{\circ}$ '08.54" S - 7540'44.46" O, $420 \mathrm{~m}$ de altitud) de la Sociedad Agrícola Drokasa (AGROKASA), con una extensión aproximada de 1100 ha (Fig. 1). Este fundo se encuentra dentro del dominio

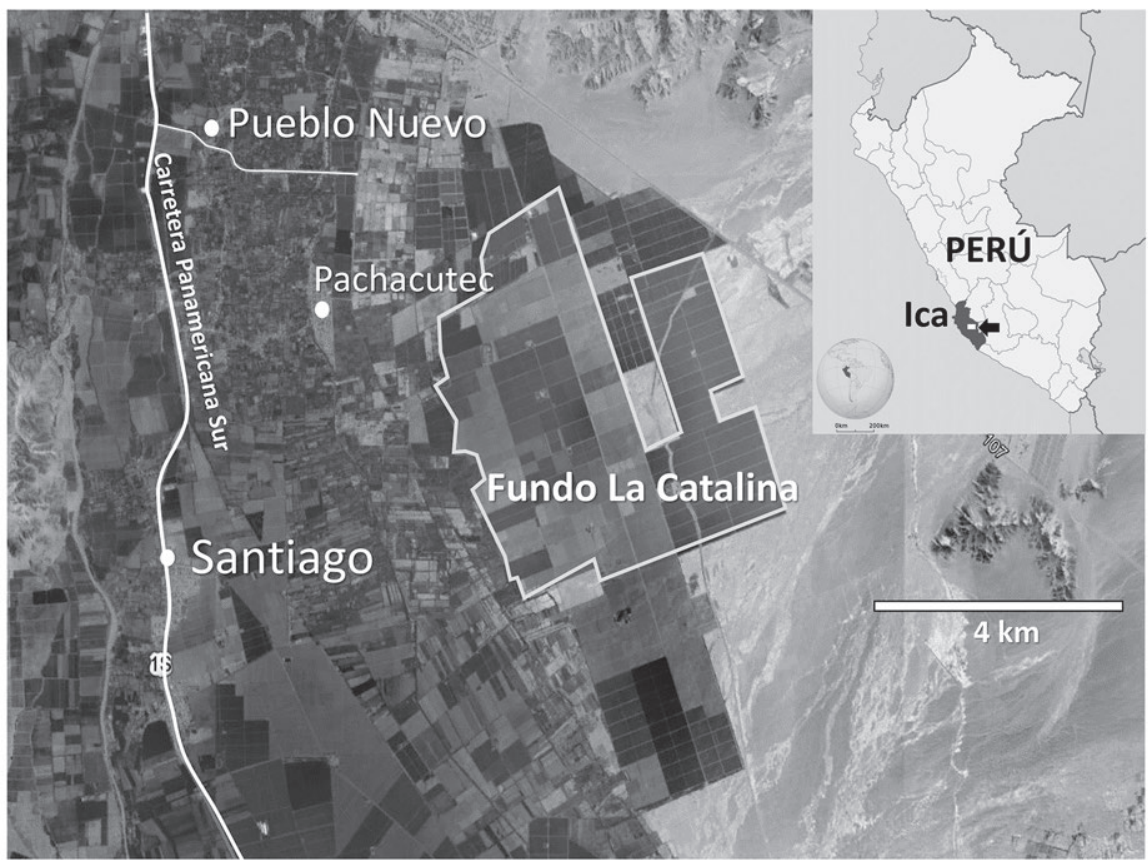

Figura 1. Ubicación del área de estudio en la región Ica: Fundo "La Catalina". 
morfoclimático del Desierto del Pacífico, caracterizado por un clima desértico cálido, con temperaturas altas durante el día, humedad relativa generalmente mayor al $60 \%$, precipitaciones poco frecuentes y vientos de moderados a fuertes (Pulido et al. $2007,2013)$. Dentro de este fundo se pueden encontrar diferentes tipos de hábitats como los cultivos de espárragos, cultivos de vid, cercos vivos de Acacia cf. horrida y Prosopis pallida; monte ribereño, y el desierto con árboles y arbustos xerofíticos (Pulido et al. 2007, Salinas et al. 2007).

Identificación de los componentes de la dieta.- Los especímenes de Zonotrichia capensis $(\mathrm{n}=133)$ y Passer domesticus ( $\mathrm{n}=113)$, fueron colectados simultáneamente en los meses de enero, abril y julio de los años 2004 a 2011. Se empleó en cada evento un mismo grupo de redes de niebla para capturar y tomar datos reproductivos y morfológicos de los individuos liberándolos posteriormente, en el presente estudio se utilizaron los contenidos estomacales sólo de los individuos que murieron accidentalmente en las redes. Los especímenes fueron trasladados al laboratorio donde se extrajeron los tractos digestivos que se fijaron en alcohol etílico $70^{\circ}$ (Salinas 2001). El contenido de cada tracto digestivo fue vaciado en placas Petri, y se separaron los diferentes componentes identificables de la dieta (semillas, restos vegetales, piedras, restos de artrópodos, entre otros), con ayuda de una lámpara con lupa.

Para identificar las semillas e insectos, se utilizó un microscopio estereoscópico para tomar fotografías y compararlas con las colecciones de referencia elaboradas con plantas e insectos colectados en el área de estudio. Adicionalmente se consultaron colecciones especializadas digitales del Royal Botanic Garden KEW, Plants Database y Seed Biology.

Análisis de los datos.- Para el análisis de los datos, se obtuvo una lista de los taxa que componen la dieta de cada gorrión. Para cada componente de la dieta se calculó el porcentaje de volumen total $(\% \mathrm{~V})$ disponiendo cada componente en la forma de un prisma rectangular y midiendo su alto, largo y ancho, también el porcentaje de abundancia de cada componente $(\% \mathrm{~N})$ es decir el número de unidades de cada componente dividido por el total de unidades de todos los componentes por cien y la frecuencia porcentual calculada como el porcentaje de contenidos estomacales que contenían cada tipo de componente dietario $(\% \mathrm{~F})$. Estos valores fueron empleados para calcular el porcentaje del Índice de importancia relativa (Pinkas et al. 1971, George \& Hadley 1979, Harrison et al. 1983, Duffy \& Jackson 1986):

$$
\text { IIR } i=(\% N+\% V) \times \% F
$$

Además se calculó la diversidad de la dieta con el índice H' de Shannon-Wiener usando logaritmo en base 2 (Magurran 2004).

Se comparó las dietas de ambos gorriones empleando los valores de volumen de los taxa consumidos, con el Índice de similitud cuantitativo de Morisita - Horn (Horn 1966) y con un análisis de escalamiento multidimensional no métrico donde se consideraron todos ítems de los contenidos estomacales colectados en el mismo grupo de redes en el mismo mes de evaluación (Kenkel \& Orlóci 1986).

Para determinar la significancia en las diferencias en los taxa consumidos por ambas especies, se empleó el test de Kolmogorov-Smirnov comparando la abundancia de cada ítem alimentario como ha sido recomendado para comparar comu- nidades y utilizado en comparación de dietas (Magurran 2004, Pérez \& Balta 2011).

El análisis de solapamiento de la dieta se realizó empleando el índice de solapamiento de Schoener $\left(\mathrm{O}_{\mathrm{jk}}\right)$, el cual es considerado como el más adecuado debido a que no requiere asunciones respecto a la naturaleza del proceso de competencia (Abrams 1980). Los valores del índice varían de 0 a 1 , valores cercanos a 0 indican bajo solapamiento, mientras que valores cercanos a 1 , indican un mayor solapamiento (Schoener 1968).

\section{Resultados}

Composición de la Dieta.- La dieta de los gorriones Zonotrichia capensis y Passer domesticus está compuesta por 27 ítems, 20 tipos de semillas y 7 de insectos (tanto adultos como larvas). Para $Z$. capensis las semillas representan $84.76 \%$ de sus ítems dietarios y los insectos el $15.24 \%$; mientras que para $P$. domesticus $70.37 \%$ y $29.63 \%$, respectivamente. La mayoría de los componentes de la dieta, fueron identificados, especialmente los más abundantes, quedando sin identificación algunos de poca abundancia, debido en gran parte a su avanzado estado de digestión (Tabla 1). Los componentes más importantes en función del \%IIR fueron las semillas de Amaranthus sp., Portulaca oleracea, Eleucine indica, Setaria verticillata y los insectos del orden coleóptera (Fig. 2).

Diversidad y solapamiento de la dieta.- El índice de Shannon-Wiener, mostró que la dieta de $Z$. capensis $\left(\mathrm{H}^{\prime}=2.07\right)$ es más diversa que la de $P$. domesticus $\left(\mathrm{H}^{\prime}=1.63\right)$. El análisis con el índice de similitud cuantitativo de Morisita-Horn, mostró un valor de $\mathrm{C}_{\mathrm{H}}=0.72$, que demuestra el elevado grado de semejanza en la dieta de ambos gorriones. La prueba de Kolmogorov-Smirnov, comprobó que los taxa que componen la dieta de los gorriones y la proporción en la que los consumen son semejantes $\left(\mathrm{D}_{\max }=\right.$ $0.15, \mathrm{p}>0.05)$.

Finalmente, la prueba de solapamiento con el índice de solapamiento de Schoener $\left(\mathrm{O}_{\mathrm{jk}}=0.73\right)$, mostró que existe un elevado grado de solapamiento. Esto mismo se observa al realizar

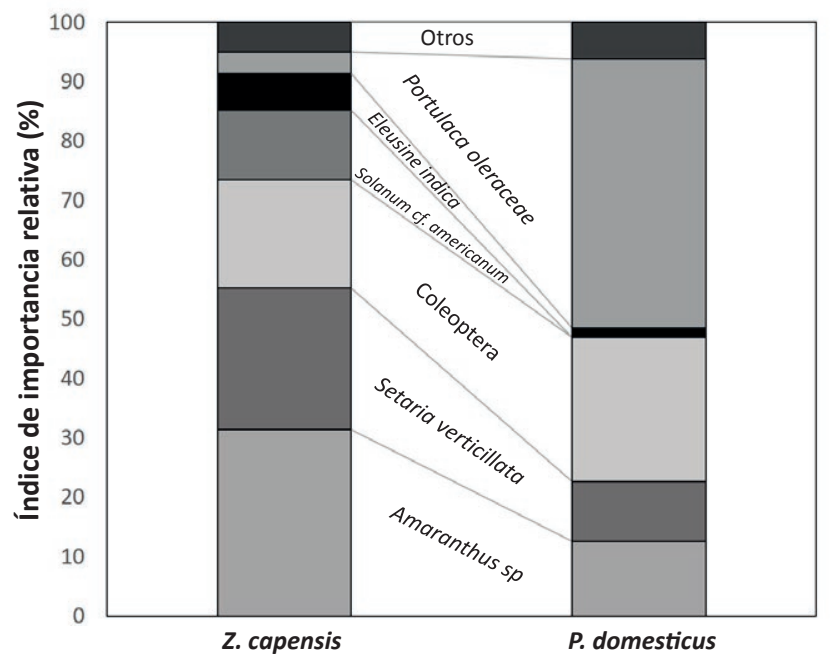

Figura 2. Comparación del porcentaje del índice de importancia relativa de los componentes más significativos de la dieta de los gorriones Zonotrichia capensis y Passer domesticus en el agroecosistema evaluado, mostrando que las especies comparten varios de ellos. 
el análisis de escalamiento multidimensional no métrico (Fig. 3) donde las dietas de las dos especies en los diferentes meses de evaluación se ordenan en prácticamente un mismo espacio y siempre se sobreponen una con la otra.

\section{Discusión}

Composición de la dieta.- El análisis inicial de la dieta de $Z$. capensis y $P$. domesticus muestra que éstas se componen mayoritariamente de semillas de malezas de los campos de cultivo y una cantidad menor de insectos. Estos datos son comparables a los obtenidos en la reserva "El Charmolán" en Colombia, donde la dieta de $Z$. capensis estuvo compuesta en más del $70 \%$ por semillas y un porcentaje cercano al 20\% de insectos (Cabrera et al. 2011). Otro estudio realizado en la Quebrada de La Plata en Chile, muestras que la dieta, estuvo compuesta de semillas que representaron entre $78 \%$ y $99 \%$ del total de alimentos consumidos, manteniendo en alrededor del $4.5 \%$ la proporción de insectos consumidos (López 1995).

Sin embargo, cuando $Z$. capensis se encuentra en otro tipo de ecosistemas, estos porcentajes varían, por ejemplo, en la isla Carabajal en Argentina, su dieta se compuso casi en la misma proporción de insectos y semillas (Alessio et al. 2005), y en la restinga del Parque Nacional Jurubatiba del Brasil, llegó a alimentarse en mayor proporción de insectos (62\%), mientras que las semillas fueron sólo el 38\% (Souza et al. 2008).

En cuanto a la dieta de $P$. domesticus, en lugares donde se encuentra naturalmente distribuido, por ejemplo, en los campos de cultivo de la Península Ibérica, las semillas representaron el

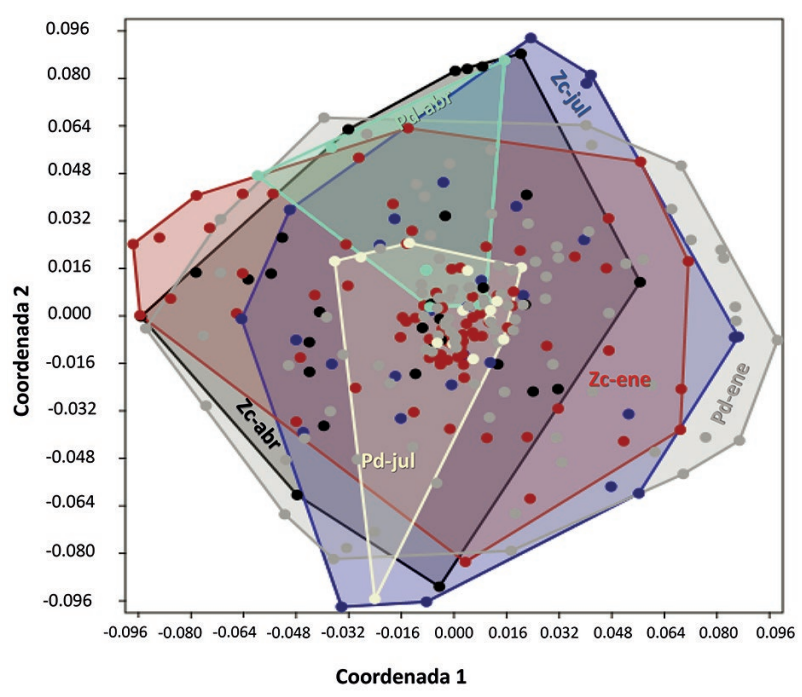

Figura 3. Análisis de escalamiento multidimensional no métrico basado en el índice de Morisita de los componentes de las dietas de Zonotrichia capensis y Passer domesticus separado por mes de colecta (Pd-ene: $P$. domesticus colectado en enero, puntos y polígono grises; Pd-abr: $P$. domesticus colectado en abril, puntos y polígono celestes; Pd-jul: $P$. domesticus colectado en julio, puntos y polígono crema; Zc-ene: Z. capensis colectado en enero, puntos y polígono marrones; Zc-abr: Z. capensis colectado en abril, puntos y polígono negros; Zc-jul: Z. capensis colectado en julio, puntos y polígono azules), mostrando el fuerte solapamiento entre ellas en todos los meses. Estrés 0.9085 .

Tabla 1. Composición de la dieta de Zonotrichia capensis y Passer domesticus, expresada en \%Volumen (\%V), \%Número $(\% \mathrm{~N})$, \%Frecuencia $(\% \mathrm{~F})$, y porcentaje del Índice de importancia relativa $(I I R=[\% N+\% V] \times \% F)$

\begin{tabular}{|c|c|c|c|c|c|c|c|c|}
\hline \multirow{2}{*}{ ITEM } & \multicolumn{4}{|c|}{ Zonotrichia capensis } & \multicolumn{4}{|c|}{ Passer domesticus } \\
\hline & $\% \mathrm{~V}$ & $\% \mathbf{N}$ & $\% \mathrm{~F}$ & $\%$ IIIR & $\% \mathrm{~V}$ & $\% \mathbf{N}$ & $\% \mathrm{~F}$ & \%IIR \\
\hline Amaranthus sp. & 17.4 & 30.5 & 14.3 & 31.45 & 7.2 & 15.5 & 13.0 & 12.71 \\
\hline Alternanthera halimifolia & 0.0 & 0.0 & 0.0 & 0.00 & 0.6 & 0.7 & 0.9 & 0.05 \\
\hline Setaria verticillata & 12.4 & 14.1 & 19.5 & 23.86 & 7.4 & 10.4 & 13.0 & 9.99 \\
\hline Eleusine indica & 3.8 & 9.8 & 9.8 & 6.11 & 1.5 & 4.7 & 5.6 & 1.50 \\
\hline Leptochloa uninervia & 0.8 & 2.6 & 3.0 & 0.47 & 1.2 & 4.6 & 1.9 & 0.47 \\
\hline Poaceae- Especie 3 & 0.1 & 0.2 & 0.8 & 0.01 & 1.0 & 2.5 & 3.7 & 0.55 \\
\hline Cynodon dactylon & 0.0 & 0.0 & 0.0 & 0.00 & 0.1 & 0.1 & 1.9 & 0.02 \\
\hline Portulaca oleracea & 2.6 & 12.5 & 5.3 & 3.66 & 9.3 & 53.6 & 16.7 & 45.36 \\
\hline Chamaesyce sp. & 2.8 & 7.1 & 3.0 & 1.37 & 0.2 & 0.7 & 1.9 & 0.08 \\
\hline Chenopodium sp. & 3.1 & 5.0 & 4.5 & 1.68 & 0.2 & 0.4 & 1.9 & 0.05 \\
\hline Chenopodiaceae Especie 1 & 1.3 & 1.8 & 1.5 & 0.21 & 0.0 & 0.0 & 0.0 & 0.00 \\
\hline Sida cf. spinosa & 0.4 & 0.2 & 1.5 & 0.04 & 0.0 & 0.0 & 0.0 & 0.00 \\
\hline Malvastrum coromandelianum & 0.4 & 0.4 & 0.8 & 0.03 & 2.6 & 3.2 & 7.4 & 1.87 \\
\hline Especie 5 & 0.4 & 0.1 & 0.8 & 0.02 & 0.7 & 0.3 & 1.9 & 0.08 \\
\hline Solanum cf. americanum & 10.6 & 13.7 & 10.5 & 11.72 & 0.0 & 0.0 & 0.0 & 0.00 \\
\hline Asteraceae Especie 2 & 1.3 & 0.4 & 0.8 & 0.06 & 0.0 & 0.0 & 0.0 & 0.00 \\
\hline Nyctaginaceae Especie 4 & 0.0 & 0.0 & 0.0 & 0.00 & 1.3 & 0.8 & 1.9 & 0.17 \\
\hline Especie 6 & 1.3 & 0.4 & 0.8 & 0.06 & 0.0 & 0.0 & 0.0 & 0.00 \\
\hline Especie 7 & 0.0 & 0.0 & 0.0 & 0.00 & 0.2 & 1.0 & 1.9 & 0.10 \\
\hline Citrus sp. & 0.0 & 0.0 & 0.0 & 0.00 & 0.5 & 0.4 & 1.9 & 0.07 \\
\hline Coleoptera & 34.8 & 0.4 & 11.3 & 18.26 & 42.8 & 0.6 & 13.0 & 24.33 \\
\hline Tenebrionidae & 3.0 & 0.1 & 3.8 & 0.55 & 2.5 & 0.1 & 3.7 & 0.42 \\
\hline Scarabidae & 1.8 & 0.1 & 2.3 & 0.20 & 2.7 & 0.2 & 4.6 & 0.58 \\
\hline Staphyllinidae & 0.6 & 0.1 & 0.8 & 0.02 & 0.0 & 0.0 & 0.0 & 0.00 \\
\hline Hymenoptera & 0.3 & 0.1 & 2.3 & 0.05 & 10.7 & 0.1 & 2.8 & 1.30 \\
\hline Formicidae & 0.9 & 0.4 & 3.0 & 0.19 & 0.0 & 0.0 & 0.0 & 0.00 \\
\hline Lepidoptera & 0.0 & 0.0 & 0.0 & 0.00 & 7.2 & 0.0 & 0.9 & 0.29 \\
\hline
\end{tabular}


95\%; mientras que los insectos sólo el 5\% (Alonso 1985); y en el río Jarama-Madrid, su dieta se compuso de $75 \%$ de semillas y 25\% de insectos (Sánchez 1986). En áreas donde P. domesticus es introducido, también muestra preferencia por las semillas; así en Punjab-India, el porcentaje de semillas fue $90 \%$, mientras que los insectos fueron apenas 5\% (Saini \& Dhindsa 1991), igualmente en Jodhpur-India, las semillas representaron entre $70 \%$ y $90 \%$ de su dieta, y los insectos entre 30\% y $10 \%$, dependiendo de las estaciones (Rana \& Idris 1987). Todas estas investigaciones coinciden en reportar la preferencia de $P$. domesticus por las semillas, resultados que coinciden con los reportados en el presente estudio.

Los presentes resultados muestran que la dieta de $Z$. capensis, está principalmente compuesta por semillas de malezas de las familias Amaranthaceae, Poaceae, Solanaceae y Portulacaceae, mientras que en los insectos destaca el orden Coleoptera. Resultados comparables fueron encontrados en estudios realizados en Colombia (Cabrera et al. 2011) y Chile (López 1995), donde se reportaron un marcado predominio de la familia Poaceae entre las semillas y del orden Coleoptera entre los insectos. Por otra parte, De la Peña \& Pensier (2003) reportan el consumo de semillas pertenecientes a las familias Chenopodiaceae y Malvaceae y los géneros Chenopodium, Sida y Eleusine. Nuevamente si comparamos los resultados de este estudio con los encontrados en ecosistemas con mayor complejidad vegetal como la restinga brasileńa, se observan diferencias notables en la dieta de $Z$. capensis, que prefiere alimentarse de las semillas de las familias Lauraceae, Burseraceae, Clusiaceae y Humiriaceae (Souza et al. 2008).

En el caso de $P$. domesticus los ítems de mayor importancia en su dieta son las semillas de las familias Portulacaceae, Amaranthaceae, Poaceae y Malvacea e insectos del orden Coleoptera. Esta composición es consistente con la reportada para este gorrión en ambientes cultivados de India (Saini \& Dhindsa 1991, Rana \& Idris 1987) y Estados Unidos (Gavett \& Wakeley 1986), donde también destacan las semillas de las familias Poaceae y Amaranthaceae, y los insectos del orden Coleoptera. Estudios realizados en España muestran que $P$. domesticus consume semillas de los géneros Chenopodium (Chenopodiaceae) y Portulaca (Portulacaceae) e insectos de los órdenes Coleoptera e Hymenoptera (Sánchez 1986, Alonso 1985), taxones también encontrados en esta investigación.

Diversidad y solapamiento de la dieta.- Los resultados de diversidad, muestran que la dieta de $Z$. capensis $\left(\mathrm{H}^{\prime}=2.07\right)$ es más diversa que la dieta de $P$. domesticus $\left(\mathrm{H}^{\prime}=1.63\right)$. Esta mayor diversidad de alimentos puede deberse a que siendo $Z$. capensis una especie nativa, aprovecharía recursos poco abundantes, mientras que el gorrión invasor, que sólo habita estos ecosistemas las últimas décadas aprovecha sólo los recursos más abundantes. La dieta de $P$. domesticus analizada en su área de distribución natural mostró valores de diversidad $\mathrm{H}^{\prime}=2.06$, en un área de cultivos cercana a Coria (Alonso 1985) y H’=3.16 en las inmediaciones de Madrid (Sánchez 1986), que son muy superiores a los encontrados en la presente investigación.

En áreas donde Passer domesticus es una especie introducida, la diversidad de su dieta baja, como en los agroecosistemas de Punjab-India, donde el índice de diversidad fue $\mathrm{H}^{\prime}=0.75$ (Saini $\&$ Dhindsa 1991). Esto podría deberse a que su dieta oportunista consiste en buscar recursos alimenticios que aporten la mayor tasa de energía, en el menor tiempo de búsqueda (Anderson 2006, Greenberg \& Mettke-Hofmann 2001).

Existen menos datos de diversidad de la dieta de $Z$. capensis. En la isla Carbajal, provincia de Santa Fe-Argentina reportaron un valor $\mathrm{H}^{\prime}=2.89$, siendo este superior al encontrado en esta investigación (Alessio et al. 2005).

El índice de Morisita - Horn $\left(\mathrm{C}_{\mathrm{H}}=0.72\right)$ muestra alta similitud en la dieta de $Z$. capensis y $P$. domesticus. Adicionalmente se conoce por De la Peña (2011) que el comportamiento alimenticio de ambas especies es semejante. Ambas recolectan alimentos en el suelo, estrato herbáceo, ramas y follaje hasta los cuatro metros, con la diferencia que $P$. domesticus caza al vuelo los insectos.

Nuestros datos muestran un solapamiento de la dieta de ambos gorriones lo que significa un peligro potencial para las poblaciones del nativo $Z$. capensis si se incrementa la población del invasor $P$. domesticus.

\section{Agradecimientos}

Al Vicerrectorado de Investigación y Posgrado de la Universidad Nacional Mayor de San Marcos, por el financiamiento de los Proyectos de Investigación 071001161, 071001291, 081001291, 091001171, 101001271, 101001281, 111001221, $111001115,121001161,121001191$. A la empresa AGROKASA por las ffacilidades brindadas en su fundo. Al SERFOR por los permisos de investigación (RD 0263-2014-MINAGRIDGFFS/DGEFFS, RD 0267-2014-MINAGRI-DGFFS/ DGEFFS, Autorización 051-2005-INRENA-IFFS-DCB). A los revisores anónimos por contribuir a mejorar el manuscrito. A Víctor Pulido, Joyce del Pino y Alejandra Arana por su ayuda en el trabajo de campo.

\section{Literatura citada}

Abrams P. 1980. Some comments on measuring niche overlap. Ecology 61(1):44-49. https://doi.org/10.2307/1937153

Alessio V., A. Beltzer, R. Lajmanovich, et al. 2005. Ecología alimentaria de algunas especies de Passeriformes (Furnariidae, Tyrannidae, Icteridae y Emberizidae): consideraciones sobre algunos aspectos del nicho ecológico. En: Aceńolaza, F., ed. Temas de biodiversidad del Litoral Fluvial Argentino II. INSUGEO Magna Publicaciones. Tucumán. Pp. 441-482.

Alonso J.C. 1985. La alimentación del gorrión común Passer domesticus en áreas de cultivo de regadío extremeńas. Ardeola 32:405-408.

Anderson T.R. 2006. Biology of the ubiquitous House Sparrow: From genes to population. Oxford University Press. New York. 560 pp.

Angulo F., T.S. Schulenberg \& E. Puse. 2010. Las aves de los humedales de Eten, Lambayeque, Perú. Ecología Aplicada 9(2):71-81. https://doi.org/10.21704/rea.v9i1-2.397

Baker M. 1995. Environmental component of latitudinal clutch-size variation in house sparrows (Passer domesticus). The Auk 112(1):249-252. https://doi.org/10.2307/4088786

Baldini A. \& L. Pancel. 2002. Agentes de daño en el bosque nativo. Editorial Universitaria. Santiago de Chile. 408 pp.

Cabrera A., Y.C. Ortiz \& J.J. Calderón. 2011. Dieta de Semilleros en un Bosque Subandino. Revista de Ciencias - Universidad de Nariño 1(1):1-8.

Chace J.F. \& J.J. Walsh. 2006. Urban effects on native avifauna: a review. Landscape and Urban Planning 74(1):46-69. https:// doi.org/10.1016/j.landurbplan.2004.08.007

De La Peña M.R. \& J.F. Pensier. 2003. Contribución de la flora en los hábitos alimentarios de las aves en un bosque del centro de la provincia de Santa Fe, Argentina. Ornitología Neotropical 14:499-513 
De La Peña M.R. 2011. Observaciones de campo en la alimentación de las aves. FAVE 15(1):1-88. https://doi.org/10.14409/ fave.v15i1.3108

Duffy D.C. \& S. Jackson. 1986. Diet studies of seabirds: a review of methods. Colonial waterbirds 9(1):1-17. https://doi. org/10.2307/1521138

Feare C. 2010. Invasive bird eradication from tropical oceanic islands. En Genovesi P. \& R. Scalera, eds. Aliens: The Invasive Species Bulletin Newsletter of the IUCN/SSC Invasive Species Specialist Group N 30. Roma. Pp. 12-19.

Gavett A.P. \& J.S. Wakeley. 1986. Diets of House Sparrows in urban and rural habitats. Wilson Bulletin 98:137-144.

George E.L. \& W.L. Hadley. 1979. Food and habitat partitioning between rock bass (Ambloplites rupestris) and smallmouth bass (Micropterus dolomievi) young of the year. Transactions of the American Fisheries Society 108(3):253-261. https://doi. org/10.1577/1548-8659(1979) 108<253:fahpbr>2.0.co;2

Goodall J.D., A.W. Johnson \& R.A. Philippi. 1946. Las aves de Chile: su conocimiento y sus costumbres. Tomo 1. Platt Establecimientos Gráficos S.A. Buenos Aires. 358 pp.

Gowaty P.A. 1981. Aggression of breeding eastern bluebirds (Sialia sialis) toward their mates and models of intra- and interspecific intruders. Animal Behaviour 29(4): 1013-1027. https://doi. org/10.1016/S0003-3472(81)80055-3

Greenberg R. \& C. Mettke-Hofmann. 2001. Ecological aspects of neophobia and neophilia in birds. Current Ornithology 16:119-178. https://doi.org/10.1007/978-1-4615-1211$0 \_3$

Gurnell J., L.A. Wauters, P.W. Lurz, et al. 2004. Alien species and interspecific competition: effects of introduced eastern grey squirrels on red squirrel population dynamics. Journal of Animal Ecology 73(1):26-35. https://doi.org/10.1111/ j.1365-2656.2004.00791.x

Harrison C.S., T.S. Hida \& M.P. Seki. 1983. Hawaiian seabird feeding ecology. Wildlife Society. California. 71pp.

Horn H.S. 1966. Measurement of "Overlap" in comparative ecological studies. The American Naturalist 100:419-424. https://doi. org/10.1086/282436

Kalinoski R. 1975. Intra and interspecific aggression in House Finches and House Sparrows. Condor 77:375-384. https://doi. org/10.2307/1366086

Kenkel N. C. \& L. Orloci. 1986. Applying Metric and Nonmetric Multidimensional Scaling to Ecological Studies: Some New Results. Ecology 67:919-928. https://doi. org/10.2307/1939814

Koepcke M. 1952. El Gorrión Europeo en el Perú. Revista Mar del Sur 22:63-72.

Koepcke M. 1964. Las Aves del Departamento de Lima. Gráfica Morson. Lima. 156 pp.

Lambertini M., J. Leape, J. Marton-Lefevre, et al. 2011. Invasives: A Major Conservation Threat. Science 333(6041): 404-405. https://doi.org/10.1126/science.333.6041.404-b

López M.V. 1995. Dieta de Zonotrichia capensis (Emberizidae) y Diuca diuca (Fringillidae): efecto de la variación estacional de los recursos tróficos y la riqueza de aves granívoras en Chile central. Revista Chilena de Historia Natural 68:321-331.

MacGregor I., L. Morales, J. Quesada \& J.E. Schondube. 2009. Relationship between the presence of House Sparrow (Passer domesticus) and Neotropical bird community structure and diversity. Biological Invasions 12:87-96. https://doi. org/10.1007/s10530-009-9432-5

Magurran A. 2004. Measuring Biological Diversity. Blackwell Publishing. Oxford. 264 pp.

Moulton M., W. Cropper, M. Avery \& L. Moulton. 2010. The earliest House Sparrow introductions to North America. Biological Invasions 12:2955-2958. https://doi.org/10.1007/s10530$010-9692-0$

Pérez J. \& K. Balta. 2011. Ecología de Phyllodactylus angustidigitus y P. gerrhopygus (Squamata: Phyllodactylidae) de la Reserva Nacional de Paracas, Perú. Revista Peruana de Biología 18(2): 217-223. https://doi.org/10.15381/rpb.v18i2.232
Pinkas L., M.S. Oliphant \& I.L.K. Iverson. 1971. Food habits of albacore Bluefin tuna and bonito in California waters. State of California, Dept. of Fish and Game. California. 105 pp.

Plants Database. 2014. Fact Sheets \& Plant Guides. (En línea). < https://plants.usda.gov/java/factSheet> Acceso 10/06/2013.

Pulido V., L. Salinas \& C. Arana. 2007. Aves en el desierto de Ica. La experiencia en AGROKASA. Editorial Biblos. Lima. 323 pp.

Pulido V., L. Salinas \& C. Arana. 2013. Aves en el desierto de la costa central del Perú. Editorial Barrón. Lima. 460 pp.

Rana D.B. \& M. Idris. 1987. Food habits of the House Sparrow Passer domesticus indicus in an arid environment. Japanese Journal of Ornithology 35:125-128.

Royal Botanic Garden KEW. Seed Information Database - SID. (En línea). < http://data.kew.org/sid/> Acceso 10/06/2013.

Saini H.K. \& M.S. Dhindsa. 1991. Diet of the house sparrow in an intensively cultivated area. Japanese Journal of Ornithology 39:93-100.

Salinas L. 2001. Aves dispersoras de semillas de plantas leñosas en el bosque de Zárate, Lima. Tesis Bióloga con mención en Zoología. EAP Ciencias Biológicas, UNMSM. Lima, 158 pp. http://sisbib-03.unmsm.edu.pe/cgi-bin/koha/opac-detail. pl?biblionumber $=74638 \#$

Salinas L. 2007. Avifauna de Tacna en la ruta del censo de suri Pterocnemia pennata. INRENA. Lima. $25 \mathrm{pp}$.

Salinas L., C. Arana \& V. Pulido. 2007. Diversidad, abundancia y conservación de aves en un agroecosistema del desierto de Ica, Perú. Revista Peruana de Biología 13(3):155-167. https://doi.org/10.15381/rpb.v13i3.2333

Salinas L., C. Arana \& A. Tello. 2013. Aves de El Olivar y otros parques de Lima. Hatch Ediciones. Lima. 118 pp.

Sánchez F.J. 1986. Sobre la alimentación de los gorriones molinero y común (Passer montanus L. y P. domesticus L.), en invierno y primavera. Ardeola 33:17-33.

Sax D. F. \& J.H. Brown. 2000. The paradox of invasion. Global Ecology and Biogeography 9(5): 363-371. https://doi. org/10.1046/j.1365-2699.2000.00217.x

Schoener T.W. 1968. The Anolis lizards of Bimini: Resource partitioning in a complex fauna. Ecology 49:704-726. https://doi. org/10.2307/1935534

Schulenberg T.S., D.F. Stotz, D.F. Lane, et al. 2010. Aves del Perú. Princeton Unity Press. Chicago. 656 pp.

Seed Biology. Seed ID Workshop. (En línea). < https://www.oardc. ohio-state.edu/seedid/> Acceso 10/06/2013

Singh R., P. Kour \& S. Koul. 2016. Comparative study of food habits and seasonal variations in the food of adult House Sparrow Passer domesticus indicus in some rural and urban areas of Jammu Province. International Journal of Advanced Research 4(7):951-958. https://doi.org/10.21474/ijar01/901

Souza V., B.A. Loiselle \& Alves M.A. 2008. Birds foraging for fruits and insects in shrubby restinga vegetation, southeastern Brazil. Biota Neotropica 8(4):21-31. https://doi.org/10.1590/ S1676-06032008000400001

Takano F. \& N. Castro. 2007. Avifauna en el campus de la Universidad Nacional Agraria la Molina (UNALM), Lima - Perú. Ecología Aplicada 6(1,2):149-154. https://doi.org/10.21704/ rea.v6i1-2.351

Torres-Vila L., J. Ferrero-García, D. Martín-Vertedor, et al. 2015. Sparrow plagues in Extremadura (Western Spain) over four centuries (1501-1900): A Spatio-temporal analysis of records from historical archives. Ardeola 62(1):19-33. https://doi.org/10.13157/arla.62.1.2015.19

Vitousek P. 1990. Biological Invasions and Ecosystem Processes: Towards an Integration of Population Biology and Ecosystem Studies. Oikos 57(1): 7-13. https://doi. org $/ 10.2307 / 3565731$

Zaret T.M. \& R.T. Paine. 1973. Species introduction in a tropical lake. Science 182(4111):449-455. https://doi.org/10.1126/ science.182.4111.449 